\title{
Anxiety disorders and age-related changes in physiology†
}

\author{
Julian Mutz, Thole H. Hoppen, Chiara Fabbri and Cathryn M. Lewis
}

\section{Background}

Anxiety disorders are leading contributors to the global disease burden, highly prevalent across the lifespan and associated with substantially increased morbidity and early mortality.

\section{Aims}

The aim of this study was to examine age-related changes across a wide range of physiological measures in middle-aged and older adults with a lifetime history of anxiety disorders compared with healthy controls.

\section{Method}

The UK Biobank study recruited $>500000$ adults, aged 37-73, between 2006 and 2010. We used generalised additive models to estimate non-linear associations between age and hand-grip strength, cardiovascular function, body composition, lung function and heel bone mineral density in a case group and in a control group.

\section{Results}

The main data-set included 332078 adults (mean age 56.37 years; $52.65 \%$ females). In both sexes, individuals with anxiety disorders had a lower hand-grip strength and lower blood pressure, whereas their pulse rate and body composition measures were higher than in the healthy control group. Case-control group differences were larger when considering individuals with chronic and/or severe anxiety disorders, and differences in body composition were modulated by depression comorbidity status.
Differences in age-related physiological changes between females in the anxiety disorder case group and healthy controls were most evident for blood pressure, pulse rate and body composition, whereas this was the case in males for hand-grip strength, blood pressure and body composition. Most differences in physiological measures between the case and control groups decreased with increasing age.

\section{Conclusions}

Findings in individuals with a lifetime history of anxiety disorders differed from a healthy control group across multiple physiological measures, with some evidence of case-control group differences by age. The differences observed varied by chronicity/severity and depression comorbidity.

\section{Keywords}

Ageing; anxiety; body composition; cardiovascular function; physiology

\section{Copyright and usage}

(C) The Author(s), 2022. Published by Cambridge University Press on behalf of the Royal College of Psychiatrists. This is an Open Access article, distributed under the terms of the Creative commons Attribution licence (https://creativecommons.org/ licenses/by/4.0/, which permits unrestricted re-use, distribution, and reproduction in any medium, provided the original work is properly cited.
Anxiety disorders are leading contributors to the global disease burden, highly prevalent across the lifespan and across nations, and associated with substantially increased morbidity and early mortality. ${ }^{1-4}$ A population-based study from Denmark reported that individuals with anxiety disorders had a $39 \%$ higher risk of premature death than the general population. ${ }^{5}$ This excess mortality does not result only from unnatural causes of death such as suicide, but also from increased rates of dementia, cardiovascular disease and other illnesses. Anxiety disorders are also associated with accelerated biological ageing, including earlier neurodegeneration $^{6,7}$ and telomere attrition, ${ }^{8,9}$ and an increased risk of disability in old age, especially in individuals with comorbid depression. ${ }^{10,11}$ Less is known about physiological differences between individuals with anxiety disorders and healthy controls, and whether such differences vary by age.

A Dutch longitudinal study reported that individuals with anxiety disorders had poorer lung function than healthy controls, and that males with anxiety disorders showed a greater decline in lung function over time. ${ }^{12}$ Females with anxiety disorders also had a lower hand-grip strength. More severe anxiety disorders were associated with greater physiological abnormalities. Most studies of physiology in individuals with anxiety disorders have

† This article has been updated since original publication and the error rectified in online PDF and HTML versions. A notice detailing the changes has also been published at https://doi.org/10.1192/bjp.2022.30. focused on one or two physiological measures ${ }^{12-14}$ and research examining a range of physiological measures is lacking.

To the best of our knowledge, this is the first study to examine age-related changes across a wide range of physiological measures in middle-aged and older adults with anxiety disorders. Most physiological measures can be assessed non-invasively, fast and at low cost, while providing reliable information on functional decline. Importantly, variation in physiological functioning predicts morbidity and mortality. A greater understanding of agerelated physiological changes in individuals with anxiety disorders may inform strategies for prevention and intervention to foster healthy ageing.

\section{Aims}

The aim of this cross-sectional study was to examine associations between age and 15 physiological markers in individuals with a lifetime history of anxiety disorders compared with healthy controls. Since the epidemiology of anxiety disorders ${ }^{2}$ and human physiology ${ }^{15}$ differ by sex, we conducted separate analyses in males and females. Given that a dose-response relationship between anxiety disorder severity and differences in physiology has been reported before, ${ }^{12}$ we also examined chronic and/or severe anxiety disorders. Finally, depression has been associated with age-related changes in physiology ${ }^{16}$ and is highly comorbid with anxiety disorders, ${ }^{2-4,12}$ hence we also examined individuals with anxiety disorders without comorbid depression. 


\section{Method}

\section{Study population}

The UK Biobank is a prospective study of $>500000$ adults aged 3773 at baseline, recruited between 2006 and 2010. The study rationale and design have been described elsewhere. ${ }^{17}$ Briefly, individuals registered with the UK National Health Service (NHS) and living within a $\sim 40 \mathrm{~km}$ radius of 1 of 22 assessment centres were invited to participate. Participants provided information on their sociodemographic characteristics, lifestyle and medical history and underwent physical examination. Hospital in-patient records are available for most participants and primary care records are currently available for half of participants. A subset of 157366 out of 339092 invited participants (46\%) completed an online follow-up mental health questionnaire (MHQ) between 2016 and 2017, covering $31 \%$ of all participants.

\section{Exposures}

Age at the baseline assessment was the primary explanatory variable. Details of the definition of participants in the case group and healthy control group are presented in Supplementary file 1 available at https://doi.org/10.1192/bjp.2021.189. Briefly, we used a transdiagnostic phenotype for lifetime anxiety disorders and identified individuals as 'cases' from multiple sources: the generalised anxiety disorder module of the Composite International Diagnostic Interview Short Form (CIDI-SF) (Supplementary file 2) ${ }^{18}$ and the Generalised Anxiety Disorder Assessment (GAD-7), ${ }^{19}$ which were assessed through the MHQ; psychiatric diagnoses reported during the nurse-led interview at baseline or in the MHQ; hospital inpatient records (Supplementary file 3); primary care records ${ }^{20}$ (Supplementary file 4). We excluded individuals with bipolar disorder or psychosis, as these disorders are strongly associated with the risk of physical multimorbidity. ${ }^{21,22}$ Individuals in the healthy control group had no anxiety disorders, reported no current psychotropic medication use at baseline (Supplementary file 5$)^{23}$ and had no other mental disorders: no psychiatric diagnosis according to the nurse-led interview, MHQ, hospital in-patient or primary care records; no probable mood disorder ${ }^{24}$ (Supplementary file 6); no Patient Health Questionnaire-9 (PHQ-9) sum score of $\geq 5$; no GAD-7 sum score of $\geq 5$; did not report ever feeling worried, tense or anxious for most of a month or longer; no depression or bipolar disorder based on the CIDI-SF depression module and questions on (hypo)manic symptoms. ${ }^{16,25}$

\section{Physiological measures}

We examined 15 physiological measures obtained at the baseline assessment, including maximal hand-grip strength, systolic and diastolic blood pressure, pulse rate, body mass index (BMI), waist-hip ratio, fat mass, fat-free mass, body fat percentage, peak expiratory flow, forced vital capacity (FVC), forced expiratory volume in $1 \mathrm{~s}\left(\mathrm{FEV}_{1}\right), \mathrm{FVC} / \mathrm{FEV}_{1}$ ratio, heel bone mineral density and arterial stiffness. Details of these measures have previously been reported in our study on age-related physiological changes in depression ${ }^{16}$ and are presented in Supplementary file 7 .

\section{Exclusion criteria}

Participants whose genetic and self-reported sex did not match and participants with missing data, including 'do not know' or 'prefer not to answer', for any covariates were excluded.

\section{Covariates}

Covariates were identified from previous studies and included ethnicity, highest educational/professional qualification, ${ }^{26}$ physical activity (walking, moderate and vigorous-intensity activity), smoking status, alcohol intake frequency, sleep duration and, for cardiovascular measures, antihypertensive medication use (UK Biobank data fields 6153 and 6177). Details of the sociodemographic and lifestyle factors are available in our previous publication. ${ }^{27}$

\section{Statistical analyses}

Analyses were prespecified prior to inspection of the data (preregistration: osf.io/hqu2f) and algorithms were tested on simulated data. Statistical analyses were conducted using R (version 3.6.0).

Sample characteristics were summarised using means and standard deviations or counts and percentages. Case-control group differences were estimated using standardised mean differences (SMD, with 95\% confidence intervals).

We examined the relationship between each physiological measure and age using generalised additive models (GAMs) with the 'mgcv' package in $\mathrm{R}^{28}$ GAMs are flexible modelling approaches that allow for the relationship between an outcome variable and a continuous exposure to be represented by a non-linear smooth curve while adjusting for covariates. This approach is useful if a linear model does not capture key aspects of the relationship between variables and attempts to achieve maximum goodnessof-fit while maintaining parsimony of the fitted curve to minimise overfitting. Smoothing parameters were selected using the restricted maximum likelihood method and we used the default option of ten basis functions to represent smooth terms. Each measure was modelled against a penalised regression spline function of age with separate smooths for the anxiety disorder case group and healthy control group.

Two models were fitted for each physiological measure in males and females separately:

(a) unadjusted model: physiological measure $\sim$ anxiety disorder + s(age, by anxiety disorder).

(b) adjusted model: physiological measure $\sim$ anxiety disorder + s(age, by anxiety disorder) + covariates (see previous section).

where s(age, by anxiety disorder) represents the smooth function for age, stratified by anxiety disorder status.

To formally test whether the relationships between physiological measures and age differed between the anxiety disorder case group and control group, we also fitted models that included reference smooths for healthy controls and difference smooths for individuals with anxiety disorders compared with healthy controls. For these analyses, anxiety disorder status was coded as an ordered factor in R. If the difference smooth differs from zero, the physiological measure follows a different trend with age in individuals with anxiety disorders and healthy controls.

Adjusted $P$-values were calculated using the $p$. adjust function in $\mathrm{R}$ to account for multiple testing across each set of analyses of the 15 physiological measures. Two methods were used: (a) Bonferroni and (b) Benjamini \& Hochberg, ${ }^{29}$ two-tailed with $\alpha=0.05$ and false discovery rate of $5 \%$, respectively. We have opted for this approach because the standard Bonferroni correction is usually too conservative, potentially leading to a high number of false negatives.

In a secondary analysis, we examined individuals with chronic and/or severe anxiety disorders, defined as individuals with:

(a) a hospital in-patient record of anxiety disorders as the primary diagnosis; 


\begin{tabular}{|c|c|c|c|c|c|c|}
\hline & \multicolumn{2}{|c|}{$\begin{array}{c}\text { Overall } \\
(n=502521)\end{array}$} & \multicolumn{2}{|c|}{ Females } & \multicolumn{2}{|c|}{ Males } \\
\hline & Mean (s.d.) & Missing, $n$ & $\begin{array}{l}\text { Healthy control } \\
\text { group, mean (s.d.) } \\
\quad(n=145364)\end{array}$ & $\begin{array}{l}\text { Anxiety disorder } \\
\text { group, mean (s.d.) } \\
\quad(n=29482)\end{array}$ & $\begin{array}{l}\text { Healthy control } \\
\text { group, mean (s.d.) } \\
(n=141992)\end{array}$ & $\begin{array}{l}\text { Anxiety disorder } \\
\text { group, mean (s.d.) } \\
\quad(n=15240)\end{array}$ \\
\hline Hand-grip strength & 31.77 (11.34) & 3203 & $24.89(6.48)$ & $24.68(6.64)$ & $41.25(9.11)$ & $40.53(9.22)$ \\
\hline Systolic blood pressure & 137.87 (18.65) & 1325 & $135.71(19.32)$ & $133.23(18.48)$ & $141.28(17.42)$ & $139.63(16.92)$ \\
\hline Diastolic blood pressure & $82.26(10.15)$ & 1323 & $80.70(9.96)$ & $80.29(9.91)$ & $84.22(9.96)$ & $83.92(9.93)$ \\
\hline Pulse rate & $69.42(11.26)$ & 1323 & $69.87(10.40)$ & 70.33 (10.68) & $67.76(11.60)$ & $68.66(12.04)$ \\
\hline Body mass index & $27.43(4.80)$ & 3105 & $26.66(4.85)$ & $26.89(5.20)$ & $27.64(4.02)$ & $27.76(4.33)$ \\
\hline Body fat percentage & $31.45(8.55)$ & 10408 & $36.06(6.74)$ & $36.40(6.96)$ & $24.97(5.63)$ & $25.27(5.81)$ \\
\hline Fat mass & $24.86(9.57)$ & 10973 & $26.14(9.50)$ & $26.83(10.23)$ & $21.87(7.88)$ & $22.37(8.48)$ \\
\hline Fat-free mass & $53.22(11.50)$ & 10176 & $44.33(4.84)$ & $44.62(5.05)$ & $63.67(7.62)$ & $63.80(7.89)$ \\
\hline Waist-hip ratio & $0.87(0.09)$ & 2265 & $0.81(0.07)$ & $0.82(0.07)$ & $0.93(0.06)$ & $0.94(0.06)$ \\
\hline \multicolumn{7}{|l|}{ Lung function ${ }^{a}$} \\
\hline Peak expiratory flow & $407.82(130.78)$ & 168566 & $352.52(76.81)$ & $356.88(77.84)$ & 510.07 (115.61) & $509.72(115.63)$ \\
\hline Forced expiratory volume $1 \mathrm{~s}$ & $2.85(0.78)$ & 149197 & $2.48(0.53)$ & $2.51(0.53)$ & $3.43(0.74)$ & $3.44(0.74)$ \\
\hline Forced vital capacity & $3.78(0.98)$ & 149197 & $3.25(0.64)$ & $3.30(0.65)$ & $4.57(0.88)$ & $4.58(0.89)$ \\
\hline $\mathrm{FEV}_{1} / \mathrm{FVC}$ & $0.75(0.07)$ & 149197 & $0.76(0.06)$ & $0.76(0.06)$ & $0.75(0.07)$ & $0.75(0.07)$ \\
\hline Heel bone mineral density ${ }^{b}$ & $0.54(0.14)$ & 180831 & $0.52(0.12)$ & $0.52(0.12)$ & $0.58(0.15)$ & $0.57(0.15)$ \\
\hline Arterial stiffness ${ }^{C}$ & $9.34(4.05)$ & 332721 & $8.72(4.27)$ & $8.71(3.33)$ & $9.94(4.59)$ & $9.94(2.92)$ \\
\hline
\end{tabular}

(b) recurrent or chronic anxiety (E2004 or E2005) in their primary care record; or

(c) generalised anxiety disorder according to the CIDI-SF with maximum level of impairment ('Impact on normal roles during worst period of anxiety' (data field 20418) = A lot) and duration ('Longest period spent worried or anxious' (data field 20420) $=$ All my life/as long as I can remember or at least 24 months).

In a sensitivity analysis, we excluded individuals with depression comorbidity from the anxiety disorder case group. ${ }^{16}$

We conducted two additional sensitivity analyses that were not pre-registered: (a) we additionally adjusted analyses of cardiovascular measures for BMI and (b) we excluded individuals with anxiety disorders who reported current use of antidepressants at baseline from the analyses of blood pressure.

\section{Ethics}

We assert that all procedures contributing to this work comply with the ethical standards of the relevant national and institutional committees on human experimentation. All procedures were approved for the UK Biobank study by the National Information Governance Board for Health and Social Care and the NHS North West Multicentre Research Ethics Committee (11/NW/0382). No project-specific ethical approval is needed. Data access permission has been granted under UK Biobank application 45514. Written informed consent was obtained from all participants.

\section{Results}

\section{Study population}

A subset of 444690 (88.49\%) participants had complete data on all covariates. After excluding participants with missing physiological data, unclear anxiety disorder status $(n=93)$ or not meeting our inclusion criteria, we retained 332078 participants in the main data-set. Subsets of 123597, 228321 and 107958 participants were included in the analyses of lung function, heel bone mineral density and arterial stiffness, respectively (Supplementary Figure 1).

\section{Participant characteristics}

The average participant age in our main data-set was 56.37 years (s.d. $=8.11$ ) and $52.65 \%$ of participants were female. Overall, $44722(13.47 \%)$ participants in this study had a lifetime history of anxiety disorders, $65.92 \%(n=29482)$ of whom were female. Descriptive statistics for the full UK Biobank and for the analytical samples stratified by sex and anxiety disorder status are presented in Table 1 (physiological measures) and Supplementary Table 4 (covariates).

\section{Case-control group differences}

Case-control group differences by sex are presented in Table 2 . Females with anxiety disorders had a lower hand-grip strength and blood pressure than the healthy control group. Their pulse rate was elevated, and they had higher values for all body composition measures and most lung function measures compared with the control group. We did not find evidence of differences in the $\mathrm{FEV}_{1}$ / FVC ratio, heel bone mineral density or arterial stiffness.

Males with anxiety disorders had a lower hand-grip strength, blood pressure and heel bone mineral density than males in the healthy control group. Their pulse rate and all body composition measures were higher than in the control group, although the difference in fat-free mass did not survive multiple testing correction. We did not find evidence of differences in lung function or arterial stiffness in males.

The largest case-control group difference was observed for systolic blood pressure ( $\mathrm{SMD}=-0.129,95 \% \mathrm{CI}-0.142$ to -0.117 , $P_{\text {Bonf. }}<0.001$ in females and SMD $=-0.095,95 \%$ CI -0.111 to $-0.078, \mathrm{P}_{\text {Bonf. }}<0.001$ in males).

\section{Chronic and/or severe anxiety}

Between $8.37 \%$ and $9.46 \%$ of females and between $9.47 \%$ and $9.99 \%$ of males in the case group had chronic and/or severe anxiety disorders (Supplementary Table 5). In females, we observed the same overall pattern of results as in the main analysis, although all observed differences were larger in magnitude. For example, the case-control group difference in body fat percentage was $\mathrm{SMD}=0.113\left(95 \%\right.$ CI 0.074-0.151, $\left.P_{\text {Bonf. }}<0.001\right)$ (Supplementary 
Table 2 Differences in physiological measures between individuals with anxiety disorders and healthy controls ${ }^{a}$

\begin{tabular}{|c|c|c|c|c|c|c|c|c|}
\hline \multirow[b]{2}{*}{ Variable } & \multicolumn{4}{|c|}{ Females } & \multicolumn{4}{|c|}{ Males } \\
\hline & SMD & $95 \% \mathrm{Cl}$ & $P_{\text {Bonf. }}$ & $P_{\mathrm{BH}}$ & SMD & $95 \% \mathrm{Cl}$ & $P_{\text {Bonf. }}$ & $P_{\mathrm{BH}}$ \\
\hline Hand-grip strength & -0.032 & -0.044 to -0.019 & $<0.001$ & $<0.001$ & -0.079 & -0.096 to -0.062 & $<0.001$ & $<0.001$ \\
\hline Systolic blood pressure & -0.129 & -0.142 to -0.117 & $<0.001$ & $<0.001$ & -0.095 & -0.111 to -0.078 & $<0.001$ & $<0.001$ \\
\hline Diastolic blood pressure & -0.041 & -0.053 to -0.028 & $<0.001$ & $<0.001$ & -0.030 & -0.046 to -0.013 & 0.007 & 0.001 \\
\hline Pulse rate & 0.045 & 0.032 to 0.057 & $<0.001$ & $<0.001$ & 0.078 & 0.061 to 0.095 & $<0.001$ & $<0.001$ \\
\hline Body mass index & 0.047 & 0.035 to 0.060 & $<0.001$ & $<0.001$ & 0.029 & 0.013 to 0.046 & 0.017 & 0.002 \\
\hline Body fat percentage & 0.050 & 0.038 to 0.063 & $<0.001$ & $<0.001$ & 0.054 & 0.037 to 0.070 & $<0.001$ & $<0.001$ \\
\hline Fat mass & 0.071 & 0.059 to 0.084 & $<0.001$ & $<0.001$ & 0.063 & 0.046 to 0.080 & $<0.001$ & $<0.001$ \\
\hline Fat-free mass & 0.058 & 0.046 to 0.071 & $<0.001$ & $<0.001$ & 0.017 & 0.000 to 0.034 & 0.831 & 0.083 \\
\hline Waist-hip ratio & 0.044 & 0.031 to 0.056 & $<0.001$ & $<0.001$ & 0.091 & 0.074 to 0.107 & $<0.001$ & $<0.001$ \\
\hline Peak expiratory flow & 0.057 & 0.035 to 0.078 & $<0.001$ & $<0.001$ & -0.003 & -0.029 to 0.024 & $>0.999$ & 0.886 \\
\hline Forced expiratory volume $1 \mathrm{~s}$ & 0.064 & 0.042 to 0.085 & $<0.001$ & $<0.001$ & 0.010 & -0.017 to 0.036 & $>0.999$ & 0.582 \\
\hline Forced vital capacity & 0.067 & 0.045 to 0.088 & $<0.001$ & $<0.001$ & 0.013 & -0.013 to 0.040 & $>0.999$ & 0.442 \\
\hline $\mathrm{FEV}_{1} / \mathrm{FVC}$ & 0.011 & -0.010 to 0.033 & $>0.999$ & 0.347 & -0.005 & -0.031 to 0.022 & $>0.999$ & 0.844 \\
\hline Heel bone mineral density & -0.005 & -0.021 to 0.010 & $>0.999$ & 0.538 & -0.067 & -0.088 to -0.047 & $<0.001$ & $<0.001$ \\
\hline Arterial stiffness & -0.003 & -0.025 to 0.018 & $>0.999$ & 0.712 & -0.001 & -0.029 to 0.027 & $>0.999$ & 0.927 \\
\hline
\end{tabular}

Table 6), compared with SMD $=0.050\left(95 \%\right.$ CI $0.038-0.063, P_{\text {Bonf. }}$ $<0.001)$ in the main analysis.

In males in the chronic and/or severe case group, we did not find evidence of a difference in diastolic blood pressure compared with healthy controls (SMD $=-0.013,95 \% \mathrm{CI}-0.064$ to $0.038, P_{\mathrm{BH}}=$ 0.713 ) and the difference in body fat percentage was not statistically significant after multiple testing correction. For most other physiological measures, we observed larger case-control group differences than in the main analysis (Supplementary Table 6). For example, the case-control group difference in systolic blood pressure was SMD $=-0.095\left(95 \%\right.$ CI -0.111 to $\left.-0.078, P_{\text {Bonf. }}<0.001\right)$ in the main analysis and $\mathrm{SMD}=-0.153(95 \% \mathrm{CI}-0.204$ to -0.102 , $\left.P_{\text {Bonf. }}<0.001\right)$ in this analysis.

\section{Anxiety without depression comorbidity}

After excluding participants in the anxiety disorder case group with depression comorbidity, we retained between $25.91 \%$ and $37.67 \%$ of females and between $31.45 \%$ and $44.69 \%$ of males with anxiety disorders (Supplementary Table 5). In females, differences in handgrip strength, blood pressure, pulse rate and arterial stiffness remained statistically significant but were smaller in magnitude than in the main analysis (Supplementary Table 7). BMI, fat mass and fat-free mass, which were higher in individuals with anxiety disorders than in healthy controls in the main analysis, were lower in the individuals with anxiety disorders without depression comorbidity (SMDs between -0.026 and -0.049 ). We did not find evidence of case-control group differences in body fat percentage or waist-hip ratio in this analysis. There was also no longer evidence of differences in lung function, except that the $\mathrm{FEV}_{1} / \mathrm{FVC}$ ratio was lower in individuals with anxiety disorders than in the control participants ( $\mathrm{SMD}=-0.048,95 \% \mathrm{CI}-0.082$ to -0.013 , $P_{\mathrm{BH}}=0.019$ ). Finally, heel bone mineral density was lower in females with anxiety disorders (SMD $=-0.036,95 \% \mathrm{CI}-0.060$ to $-0.012, P_{\text {Bonf. }}=0.043$ ).

In males, differences in hand-grip strength, blood pressure, pulse rate, waist-hip ratio, lung function, heel bone mineral density and arterial stiffness were similar to the main analysis. BMI and fat-free mass were lower in individuals with anxiety disorders than in the control participants $(\mathrm{SMD}=-0.058,95 \% \mathrm{CI}$ -0.084 to $-0.033, P_{\text {Bonf. }}<0.001$ and $\mathrm{SMD}=-0.066,95 \% \mathrm{CI}$ -0.092 to $-0.041, P_{\text {Bonf. }}<0.001$, respectively) and we did not find evidence of case-control group differences in body fat percentage or fat mass, which were elevated in the case group in the main analysis.

\section{Case-control group differences by age}

We found some evidence that age-related changes in blood pressure, pulse rate, body composition and heel bone mineral density differed between females in the case and control groups (Fig. 1). Systolic blood pressure was $-0.9 \mathrm{mmHg}$ lower in individuals with anxiety disorders at age 45 and this difference widened to $-2.2 \mathrm{mmHg}$ at age 65. For diastolic blood pressure, we did not find evidence of case-control group differences below age 52, and slightly lower diastolic blood pressure in the case group than in the control group above age 52 years. Case-control group differences in pulse rate and body composition narrowed with age (Supplementary Fig. 2). Heel bone mineral density was slightly lower in the case group than in the control group below age 55, and there was no evidence of differences between older people in the case and control groups.

In males, case-control group differences in hand-grip strength, pulse rate, waist-hip ratio and heel bone mineral density narrowed with age (Fig. 2 and Supplementary Fig. 3). There was some evidence that diastolic blood pressure was lower in the case group than in the control group above age 50 years, although the formal statistical test did not survive multiple testing correction. We found little evidence of case-control group differences by age for the other physiological measures.

In females, we observed similar results across all physiological measures after adjustment for covariates (Supplementary Figs 4 and 5). The formal statistical tests provided no evidence of casecontrol group differences in age-related changes in heel bone mineral density, however, the overall pattern of results was comparable with the unadjusted model. In males, we also observed similar results in the adjusted model (Supplementary Figs 6 and 7). Although the trajectories were similar to the unadjusted analysis, the formal statistical tests provided no evidence of case-control group differences in age-related changes in waist-hip ratio. The same was true for diastolic blood pressure and heel bone mineral density after multiple testing correction.

\section{Chronic and/or severe anxiety}

In females with chronic and/or severe anxiety, we observed similar results for age-related changes in blood pressure and body composition, except that there was no evidence of case-control group differences in fat-free mass below age 45 (Supplementary Figs 8 and 9). As 

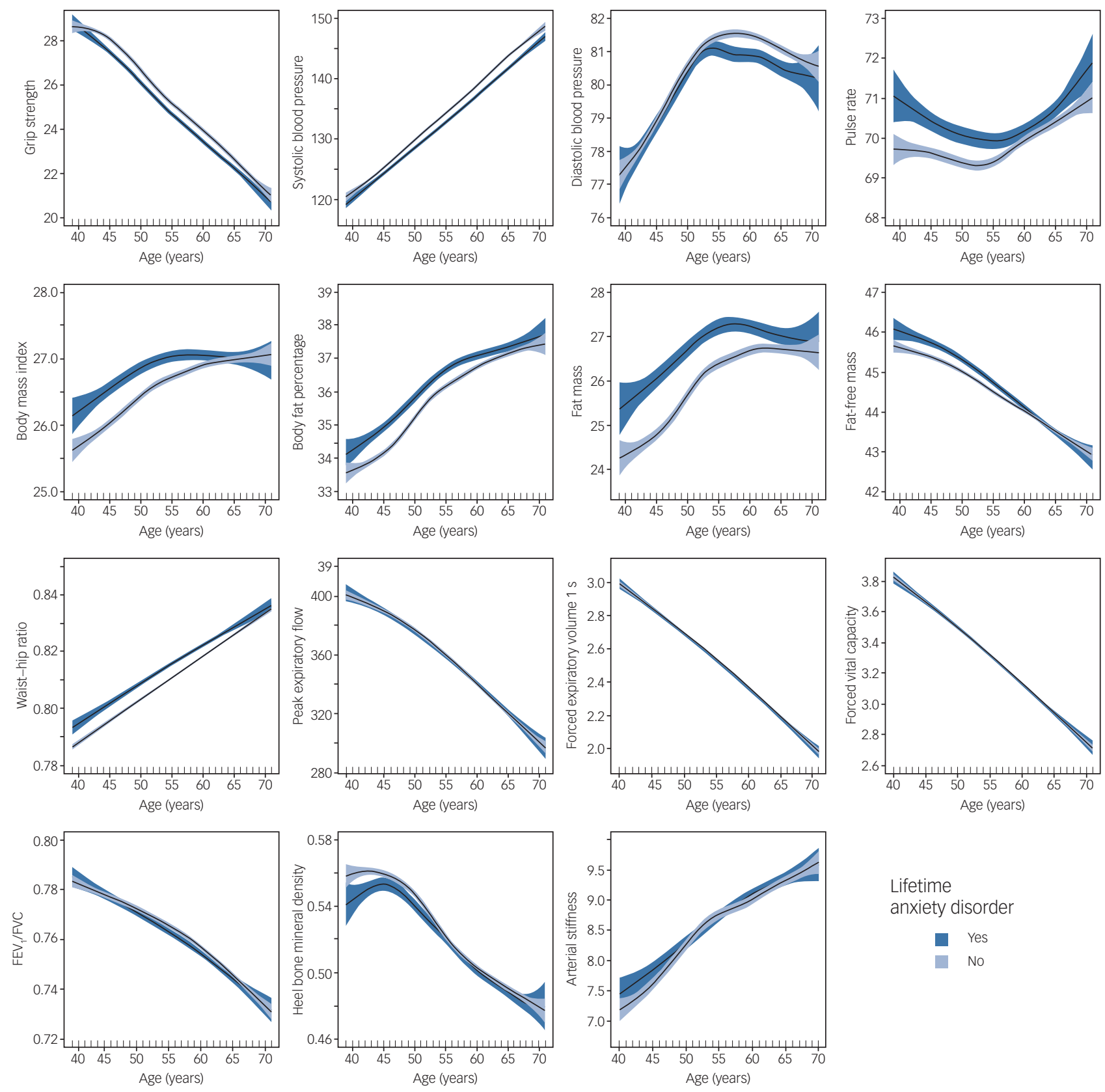

Lifetime anxiety disorder

$$
\begin{aligned}
& \text { Yes } \\
& \text { No }
\end{aligned}
$$

\section{Fig. 1 Generalised additive models of age-related changes in physiological measures in females with anxiety disorders and healthy controls}

The solid lines represent physiological measures against smoothing functions of age. The shaded areas correspond to approximate $95 \%$ confidence intervals (plus or minus $2 \times$ standard error). $\mathrm{FEV}_{1}$, forced expiratory volume in $1 \mathrm{~s}$; FVC, forced vital capacity.

in the main analysis, we observed no evidence of case-control group differences in age-related changes in lung function or arterial stiffness. Differences in results were most evident for hand-grip strength and pulse rate, and to a lesser extent for heel bone mineral density. The formal statistical tests provided some evidence of case-control group differences in age-related changes in blood pressure, BMI, fat mass and fat-free mass, although none survived multiple testing correction.

For males with chronic and/or severe anxiety, we found some evidence that case-control group differences in hand-grip strength and pulse rate narrowed with age, similar to the results from the main analysis, although none of the formal statistical tests survived multiple testing correction. There was less evidence of case-control group differences in age-related changes in systolic blood pressure and no evidence of case-control group differences in age-related changes in diastolic blood pressure. For all other physiology measures, none of the formal statistical tests were statistically significant (Supplementary Figs 10 and 11).

\section{Anxiety without depression comorbidity}

In females in the case group without depression comorbidity, none of the formal statistical tests provided evidence of case-control group differences in age-related changes in physiology. We also observed less evidence of differences by age in blood pressure or pulse rate. There was some evidence that several body composition and lung function measures were lower in individuals with anxiety disorders than in healthy controls between the ages 45 to 65 (Supplementary Figs 12 and 13). In males, the formal statistical test provided some evidence of case-control group differences in 

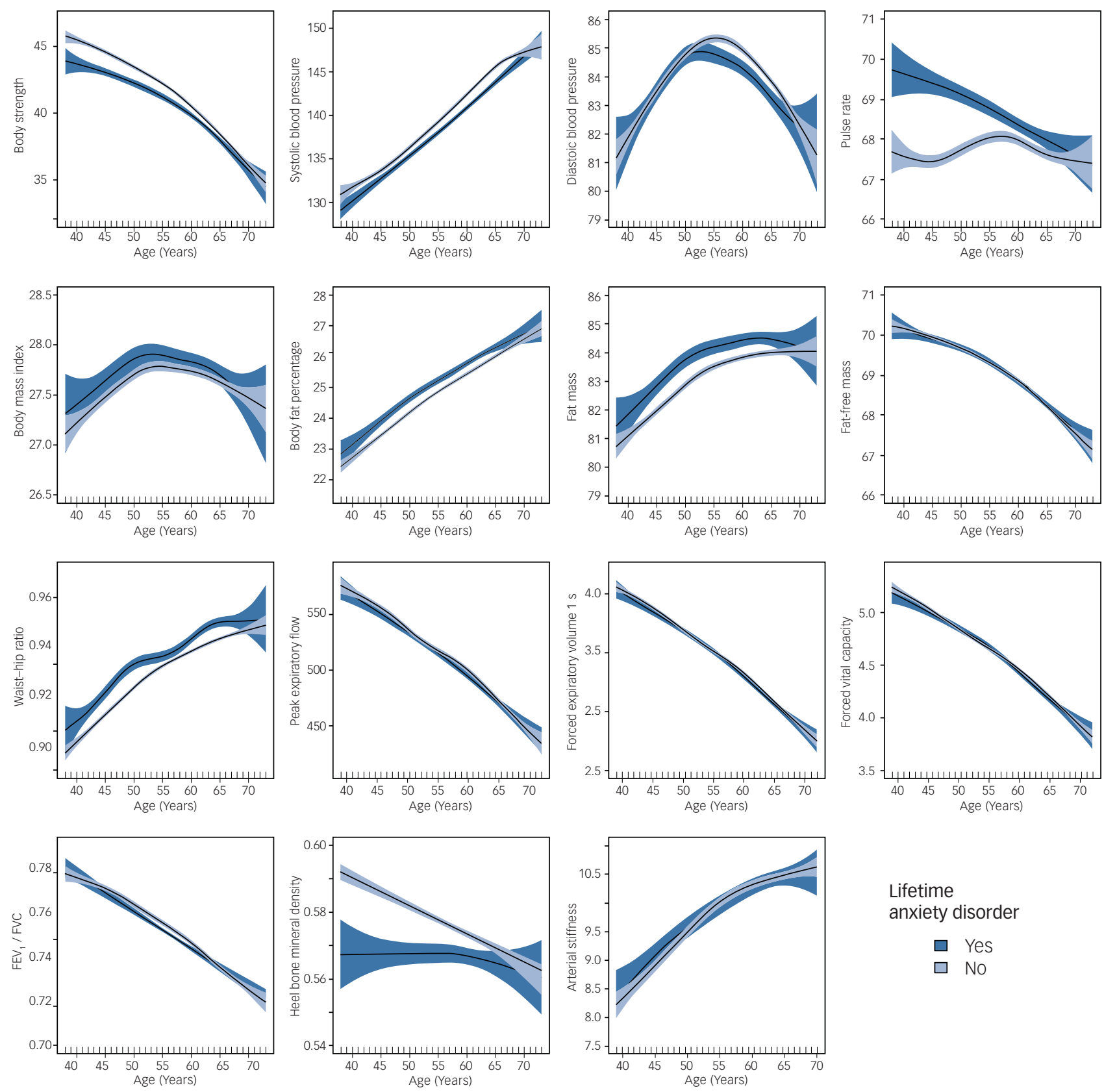

Lifetime anxiety disorder

$\square$ Yes

$\square$ No

Fig. 2 Generalised additive models of age-related changes in physiological measures in males with anxiety disorders and healthy controls.

The solid lines represent physiological measures against smoothing functions of age. The shaded areas correspond to approximate $95 \%$ confidence intervals (plus or minus $2 \times$ standard error). FEV 1 , forced expiratory volume in $1 \mathrm{~s}$; FVC, forced vital capacity.

age-related changes in pulse rate, although it did not survive multiple testing correction. None of the other formal statistical tests were statistically significant and there was less evidence of casecontrol group differences in age-related physiological changes (Supplementary Figs 14 and 15).

\section{Additional sensitivity analyses}

Case-control group differences in cardiovascular function by age were similar to the results of our main analysis after additional adjustment for BMI (Supplementary Figs 16 and 17). Excluding individuals with anxiety disorders who reported current use of antidepressants ( $n=6517$ females and $n=2700$ males) had a negligible effect on case-control group differences in blood pressure (Supplementary Table 7).

\section{Discussion}

\section{Principal findings}

We observed case-control group differences in hand-grip strength, blood pressure, pulse rate and body composition in both sexes, whereas case-control group differences in lung function and heel bone mineral density were specific to females and males, respectively. We found no evidence of case-control group differences in arterial stiffness. 
Most of the observed differences were larger when we examined participants with chronic and/or severe anxiety disorders. However, in males the difference in diastolic blood pressure was smaller and not statistically significant, and differences in body fat percentage and heel bone mineral density, although similar in magnitude, were no longer statistically significant.

After excluding individuals with anxiety disorders with comorbid depression, most differences remained statistically significant but were smaller in magnitude. However, body composition measures in both sexes were either lower in the case group, or we did not find evidence of case-control group differences. We found some evidence that heel bone mineral density was lower in females with anxiety disorders without comorbid depression. Most casecontrol group differences in lung function, however, were no longer statistically significant. We also did not find evidence of case-control group differences in diastolic blood pressure in males without comorbid depression.

Differences in age-related physiological changes between females with anxiety disorders and healthy control group were most evident for blood pressure, pulse rate and body composition, with some evidence of differences in heel bone mineral density. Most case-control group differences narrowed with age, except that we found a larger difference in blood pressure in older participants. In males, case-control group differences in hand-grip strength, pulse rate and to a lesser extent in waist-hip ratio and heel bone mineral density narrowed with age. Diastolic blood pressure was lower in older individuals with anxiety disorders than in controls.

The overall pattern of results was comparable in females with chronic and/or severe anxiety, but there was generally less evidence of differences between trajectories in males. Except for body composition, there was limited evidence of case-control group differences in age-related physiological changes after excluding individuals with comorbid depression.

\section{Findings in context}

Consistent with findings from the Netherland Study of Depression and Anxiety (NESDA), ${ }^{12}$ females with anxiety disorders had a lower hand-grip strength. Hand-grip strength was also lower in males with anxiety disorders, which had not been observed in the NESDA.

Previous findings regarding anxiety disorders and blood pressure have been mixed and studies have often examined hypertension instead of blood pressure. ${ }^{30-33}$ Although a recent metaanalysis reported increased rates of hypertension in participants with anxiety disorders, ${ }^{14}$ several studies found no statistically significant associations with hypertension or blood pressure. ${ }^{34-38}$ Some studies observed lower blood pressure in participants with anxiety disorders. ${ }^{39-41}$ Antidepressant medication and benzodiazepine use may affect blood pressure, ${ }^{42-44}$ and some populationbased studies have observed lower blood pressure in participants with depression. ${ }^{16,42}$ The high degree of comorbidity between anxiety disorders and depression and differences in medication use might partially explain these mixed results. We observed lower blood pressure in people with anxiety disorders, except for diastolic blood pressure in males, irrespective of depressive comorbidity. Excluding individuals who reported antidepressant use at baseline resulted in a negligible decrease in the case-control group difference in systolic blood pressure and a negligible increase in the difference in diastolic blood pressure. Case-control group differences in blood pressure were larger in participants with chronic and/or severe anxiety disorders.

Consistent with previous research, ${ }^{45,46}$ we observed a higher pulse rate in individuals with anxiety disorders. Noteworthy, reductions in anxiety disorder severity following cognitive-behavioural therapy have been associated with a decrease in resting pulse rate. $^{47}$

Previous research has found higher rates of obesity ${ }^{48,49}$ and poor diet ${ }^{50}$ in people with anxiety disorders, consistent with our observation that individuals with anxiety disorders had elevated measures of body composition. However, our analyses suggested that these differences may be modified by depression comorbidity. Depression has been associated with increased metabolic risk factors ${ }^{51}$ and elevated body composition measures. ${ }^{16}$ One study found that depression, but not anxiety disorders, was associated with an increased risk of metabolic syndrome, ${ }^{52}$ although a large Finnish birth cohort study found no evidence that either depression or anxiety disorders were associated with metabolic syndrome. ${ }^{53}$

Our lung function results contradict previous research. A crosssectional analysis of the NESDA data found poorer lung function in females with anxiety disorders and/or depression compared with healthy controls and better lung function in males in the case group than in the control group. ${ }^{54}$ A 6-year longitudinal assessment of these participants suggested a greater decline in lung function in men in the case group compared with the control group. ${ }^{12} \mathrm{We}$ observed better lung function in females with anxiety disorders and found no statistically significant case-control group differences in males. However, we observed no evidence of differences after excluding individuals with comorbid depression. Noteworthy, the results from the NESDA were not reported separately for anxiety disorders and depression.

Our results confirm previous research ${ }^{55}$ that found a lower bone mineral density in males with anxiety disorders and limited evidence of case-control group difference in females.

We did not observe any differences in arterial stiffness between the case and control groups. This finding is surprising given that previous studies have reported increased arterial stiffness in participants with anxiety disorders. ${ }^{56,57}$

Inconsistencies in findings between studies could result from differences in sample characteristics such as age, sex, severity of symptoms or the prevalence of effect modifiers. Systematic reviews of studies examining each measure of physiological function in relation to anxiety disorders could address these important questions. The definition of anxiety disorders, including which specific diagnoses were included, might also contribute to differences in results.

To our knowledge, we report the first study of age-related changes in physiology in people with anxiety disorders with age as a continuous rather than a categorical variable. Previous studies dichotomised age, for example testing differences between middle-aged participants and others, ${ }^{14}$ making comparisons with our study difficult.

\section{Mechanisms}

Several mechanisms could explain the physiological differences between the anxiety disorders group and the healthy control group. Anxiety disorders are sometimes associated with less healthy lifestyle behaviours ${ }^{58}$ that could affect a range of physiological makers. Physiological differences could also reflect the cumulative effects of anxiety-related overactivation of the hypothalamic-pituitary-adrenal axis and sympathetic nervous system $^{33}$ as well as increased inflammation and oxidative stress. $^{59-61}$ It is also possible that the reciprocal relationship between late-life anxiety and associated cognitive impairment may be a driver of poor physiological function. ${ }^{62}$ The greater case-control group differences observed in people with chronic and/or severe anxiety disorders could be explained by a doseresponse relationship between severity of symptoms and physiology mediated by a greater impact of anxiety on lifestyle, overactivation of the sympathetic nervous system and other potential pathways. A potential explanation for the observation that case-control group 
differences decreased with increasing age is that the prevalence of comorbidities increases with age, which may dilute the effects that anxiety disorders may have.

\section{Limitations}

The cross-sectional study design presents uncertainty about whether case-control group differences by age represent changes because of ageing or potential cohort effects. Future work should examine physiological function in anxiety disorders longitudinally. Although we found that all physiological measures varied by age, selection bias resulting in healthier older adults participating at higher rates relative to their age group could result in the underestimation of age-related changes. To achieve maximum cohort coverage, we identified individuals with anxiety disorders from multiple data sources, with strengths and limitations that have been discussed elsewhere. ${ }^{23,63,64}$ For the primary care data, individuals were included in the case group only if they had at least two mentions of anxiety in their records. Anxiety disorder case participants in this study also included individuals with a single episode of anxiety, which likely resulted in an underestimation of casecontrol group differences. A small number of individuals with subthreshold disorders could be present among the healthy controls, which could have attenuated observed differences. We examined a transdiagnostic definition of anxiety disorders, and differences in physiological function between specific diagnoses could be explored in future studies. Although we found that the differences between individuals with chronic and/or severe anxiety disorders and controls were larger, these differences could at least partly reflect higher levels of comorbidities in addition to the effects of chronicity and/or severity.

Some caution is warranted in interpreting the findings of the sensitivity analyses because of the smaller sample size and lower statistical power. Finally, limitations in the assessment of physiological function may have masked some differences between the case and control groups. For example, dynamic measures such as pulse rate were not measured longitudinally, and our study does therefore not provide insights into potential case-control group differences that could be identified from time-series data. Similarly, lung function was estimated through volumetric measures using breath spirometry and we could therefore not examine irregularity of respiratory patterns. Physiological measures with higher temporal resolution could be examined in future studies.

\section{Generalisability}

UK Biobank participants are not fully representative of the UK population. MHQ respondents were also more educated, of higher socioeconomic status and had fewer long-standing illnesses than participants who did not complete the MHQ. Similar patterns of disease prevalence were present in the MHQ and hospital in-patient records, although anxiety was reported more frequently in the MHQ. ${ }^{18}$ Nevertheless, the overall number of individuals with a lifetime history of anxiety disorders identified in our study was comparable with previous epidemiological studies. ${ }^{65}$ Wider issues of generalisability of findings from the UK Biobank have been discussed elsewhere. ${ }^{66}$ Our findings do not generalise to populations below age 40 or older than age 70 , and there was greater uncertainty near the lower and upper extremes of the age range in this study. Additional studies in younger participants and in elderly people are needed.

\section{Implications}

Individuals with a lifetime history of anxiety disorders differed from healthy controls across multiple physiological measures, with some evidence of case-control group differences by age. The differences observed varied by chronicity/severity of anxiety and depression comorbidity. Monitoring of physiological function in individuals with anxiety disorders should be adapted depending on depression comorbidity status.

Julian Mutz (D), Social, Genetic and Developmental Psychiatry Centre, Institute of Psychiatry, Psychology \& Neuroscience, King's College London, UK; Thole H. Hoppen, Institute of Psychology, University of Münster, Germany; Chiara Fabbri (D), Social, Genetic and Developmental Psychiatry Centre, Institute of Psychiatry, Psychology \& Neuroscience, King's College London, UK; and Department of Biomedical and Neuromotor Sciences, University of Bologna, Italy; Cathryn M. Lewis, Social, Genetic and Developmental Psychiatry Centre, Institute of Psychiatry, Psychology \& Neuroscience, King's College London, UK; Department of Medical and Molecular Genetics, Faculty of Life Sciences \& Medicine, King's College London, UK

Correspondence: Julian Mutz. Email: julian.mutz@gmail.com

First received 14 Aug 2021, final revision 29 Oct 2021, accepted 28 Nov 2021

\section{Supplementary material}

To view supplementary material for this article, please visit https://doi.org/10.1192/bjp.2021.189.

\section{Data availability}

The data used are available to all bona fide researchers for health-related research that is in the public interest, subject to an application process and approval criteria. Study materials are publicly available online at http://www.ukbiobank.ac.uk.

\section{Acknowledgments}

This research has been conducted using data from UK Biobank, a major biomedical database. This project made use of time on Rosalind HPC, funded by Guy's \& St Thomas' Hospital NHS Trust Biomedical Research Centre (GSTT-BRC), South London \& Maudsley NHS Trust Biomedical Research Centre (SLAM-BRC), and Faculty of Natural Mathematics \& Science (NMS) at King's college London.

\section{Author contributions}

J.M. conceived the idea of the study, acquired the data, carried out the statistical analysis, interpreted the findings, wrote the manuscript and revised the manuscript for final submission. T.H. H. contributed to the study design, interpreted the findings and contributed to the writing of the manuscript. C.F. interpreted the findings and critically reviewed the manuscript. C.M.L. acquired the studentship funding, interpreted the findings and critically reviewed the manuscript. All authors read and approved the final manuscript. J.M. had full access to all data used in this study and takes responsibility for the integrity of the data and the accuracy of the data analysis.

\section{Funding}

J.M. receives studentship funding from the Biotechnology and Biological Sciences Research Council (BBSRC) (ref: 2050702) and Eli Lilly and Company Limited. C.F. was supported by Fondazione Umberto Veronesi (https://www.fondazioneveronesi.it). C.M.L. is part-funded by the National Institute for Health Research (NIHR) Maudsley Biomedical Research Centre at South London and Maudsley NHS Foundation Trust and King's College London. The views expressed are those of the authors and not necessarily those of the NHS, the NIHR or the Department of Health and Social Care.

\section{Declaration of interest}

J.M. receives studentship funding from the Biotechnology and Biological Sciences Research Council (BBSRC) and Eli Lilly and Company Limited. C.F. has been a speaker for Janssen. C.M.L. is a member of the Scientific Advisory Board of Myriad Neuroscience. T.H.H. declares no relevant conflict of interest.

\section{References}

1 Vos T, Lim SS, Abbafati C, Abbas KM, Abbasi M, Abbasifard M, et al. Global burden of 369 diseases and injuries in 204 countries and territories, 19902019: a systematic analysis for the Global Burden of Disease Study 2019. Lancet 2020; 396: 1204-22.

2 Baxter AJ, Scott K, Vos T, Whiteford H. Global prevalence of anxiety disorders: a systematic review and meta-regression. Psychol Med 2013; 43: 897-910. 
3 Hovenkamp-Hermelink JH, Jeronimus BF, Myroniuk S, Riese H, Schoevers RA. Predictors of persistence of anxiety disorders across the lifespan: a systematic review. Lancet Psychiatry 2021; 8: 428-43.

4 Markkula N, Härkänen T, Perälä J, Partti K, Pena S, Koskinen S, et al. Mortality in people with depressive, anxiety and alcohol use disorders in Finland. $\mathrm{Br} \mathrm{J}$ Psychiatry 2012; 200: 143-9.

5 Meier SM, Mattheisen M, Mors O, Mortensen PB, Laursen TM, Penninx BW. Increased mortality among people with anxiety disorders: total population study. Br J Psychiatry 2016; 209: 216-21.

6 Perna G, Iannone G, Alciati A, Caldirola D. Are anxiety disorders associated with accelerated aging? A focus on neuroprogression. Neural Plast 2016; 2016: 8457612

7 Karim HT, Ly M, Yu G, Krafty R, Tudorascu DL, Aizenstein HJ, et al. Aging faster: worry and rumination in late life are associated with greater brain age. Neurobiol Aging 2021; 101: 13-21.

8 Malouff JM, Schutte NS. A meta-analysis of the relationship between anxiety and telomere length. Anxiety Stress Coping 2017; 30: 264-72.

9 Verhoeven JE, Révész D, van Oppen P, Epel ES, Wolkowitz OM, Penninx BW. Anxiety disorders and accelerated cellular ageing. Br J Psychiatry 2015; 206 $371-8$

10 Brenes GA, Guralnik JM, Williamson JD, Fried LP, Simpson C, Simonsick EM, et al. The influence of anxiety on the progression of disability. J Am Geriatr SoC 2005; 53: 34-9.

11 Brenes GA, Penninx BW, Judd PH, Rockwell E, Sewell DD, Wetherell JL. Anxiety, depression and disability across the lifespan. Aging Ment Health 2008; 12 $158-63$.

12 Lever-van Milligen BA, Lamers F, Smit JH, Penninx BW. Six-year trajectory of objective physical function in persons with depressive and anxiety disorders. Depress Anxiety 2017; 34: 188-97.

13 Goodwin RD, Chuang S, Simuro N, Davies M, Pine DS. Association between lung function and mental health problems among adults in the United States: findings from the First National Health and Nutrition Examination Survey. Am J Epidemiol 2007; 165: 383-8.

14 Pan Y, Cai W, Cheng Q, Dong W, An T, Yan J. Association between anxiety and hypertension: a systematic review and meta-analysis of epidemiological studies. Neuropsychiatr Dis Treat 2015; 11: 1121-30.

15 Austad SN, Bartke A. Sex differences in longevity and in responses to antiaging interventions: a mini-review. Gerontology 2016; 62: 40-6.

16 Mutz J, Lewis CM. Lifetime depression and age-related changes in body composition, cardiovascular function, grip strength and lung function: sexspecific analyses in the UK Biobank. Aging 2021; 13: 17038-79.

17 Bycroft C, Freeman C, Petkova D, Band G, Elliott LT, Sharp K, et al. The UK Biobank resource with deep phenotyping and genomic data. Nature 2018; 562 203-9.

18 Davis KA, Coleman JR, Adams M, Allen N, Breen G, Cullen B, et al. Mental health in UK Biobank-development, implementation and results from an online questionnaire completed by 157366 participants: a reanalysis. BJPsych Open 2020; 6: E18

19 Plummer F, Manea L, Trepel D, McMillan D. Screening for anxiety disorders with the GAD-7 and GAD-2: a systematic review and diagnostic metaanalysis Gen Hosp Psychiatry 2016; 39: 24-31.

20 Fabbri C, Hagenaars SP, John C, Williams AT, Shrine N, Moles L, et al. Genetic and clinical characteristics of treatment-resistant depression using primary care records in two UK cohorts. Mol Psychiatry 2021; 26: 3363-3373.

21 Stubbs B, Koyanagi A, Veronese N, Vancampfort D, Solmi M, Gaughran F, et al. Physical multimorbidity and psychosis: comprehensive cross sectional analysis including 242,952 people across 48 low-and middle-income countries. BMC Med 2016; 14: 1-12.

22 Smith DJ, Martin D, McLean G, Langan J, Guthrie B, Mercer SW. Multimorbidity in bipolar disorder and undertreatment of cardiovascular disease: a cross sectional study. BMC Med 2013; 11: 1-11.

23 Davis KA, Cullen B, Adams M, Brailean A, Breen G, Coleman JR, et al. Indicators of mental disorders in UK Biobank-A comparison of approaches. Int J Methods Psychiatr Res 2019; 28: e1796.

24 Smith DJ, Nicholl BI, Cullen B, Martin D, Ul-Haq Z, Evans J, et al. Prevalence and characteristics of probable major depression and bipolar disorder within UK biobank: cross-sectional study of 172,751 participants. PLOS One 2013; 8 : e75362.

25 Mutz J, Young AH, Lewis CM. Age-related changes in physiology in individuals with bipolar disorder. J Affect Disord 2021; 296: 157-168.

26 Guggenheim JA, Williams C. Childhood febrile illness and the risk of myopia in UK Biobank participants. Eye 2016; 30: 608-14.

27 Mutz J, Roscoe CJ, Lewis CM. Exploring health in the UK Biobank: associations with sociodemographic characteristics, psychosocial factors, lifestyle and environmental exposures. BMC Med 2021; 19: 240
28 Wood SN. Generalized Additive Models: An Introduction with R. CRC Press, 2017.

29 Benjamini Y, Hochberg Y. Controlling the false discovery rate: a practical and powerful approach to multiple testing. J R Stat Soc Series B Methodol 1995; 57: 289-300.

30 Byrd JB, Brook RD. Anxiety in the "age of hypertension". Curr Hypertens Rep 2014; 16: 1-7.

31 Cuffee Y, Ogedegbe C, Williams NJ, Ogedegbe G, Schoenthaler A. Psychosocial risk factors for hypertension: an update of the literature. Curr Hypertens Rep 2014; 16: 483

32 Player MS, Peterson LE. Anxiety disorders, hypertension, and cardiovascular risk: a review. Int J Psychiatry Med 2011; 41: 365-77.

33 Cohen BE, Edmondson D, Kronish IM. State of the art review: depression stress, anxiety, and cardiovascular disease. Am J Hypertens 2015; 28 1295-302.

34 Jackson CA, Pathirana T, Gardiner PA. Depression, anxiety and risk of hypertension in mid-aged women: a prospective longitudinal study. J Hypertens 2016; 34: 1959-66.

35 Jones-Webb R, Jacobs DR Jr, Flack JM, Liu K. Relationships between depressive symptoms, anxiety, alcohol consumption, and blood pressure: results from the CARDIA study. Alcohol Clin Exp Res 1996; 20: 420-7.

36 Maatouk I, Herzog W, Böhlen F, Quinzler R, Löwe B, Saum K-U, et al. Association of hypertension with depression and generalized anxiety symptoms in a large population-based sample of older adults. J Hypertens 2016; 34 1711-20.

37 Shinn EH, Poston WSC, Kimball KT, St. Jeor ST, Foreyt JP. Blood pressure and symptoms of depression and anxiety: a prospective study. Am J Hypertens 2001; 14: 660-4.

38 Yan LL, Liu K, Matthews KA, Daviglus ML, Ferguson TF, Kiefe Cl. Psychosocial factors and risk of hypertension: the Coronary Artery Risk Development in Young Adults (CARDIA) study. JAMA 2003; 290: 2138-48.

39 Huang $Y$, Su Y, Jiang Y, Zhu M. Sex differences in the associations between blood pressure and anxiety and depression scores in a middle-aged and elderly population: the Irish Longitudinal Study on Ageing (TILDA). J Affect Disord 2020; 274: $118-25$

40 Hildrum B, Romild U, Holmen J. Anxiety and depression lowers blood pressure: 22-year follow-up of the population based HUNT study, Norway. BMC Public Health 2011; 11: 1-8.

41 Bhat SK, Beilin LJ, Robinson M, Burrows S, Mori TA. Relationships between depression and anxiety symptoms scores and blood pressure in young adults. J Hypertens 2017; 35: 1983-91.

42 Licht CM, De Geus EJ, Seldenrijk A, Van Hout HP, Zitman FG, Van Dyck R, et al. Depression is associated with decreased blood pressure, but antidepressant use increases the risk for hypertension. Hypertension 2009; 53: 631-8.

43 Zhong Z, Wang L, Wen X, Liu Y, Fan Y, Liu Z. A meta-analysis of effects of selective serotonin reuptake inhibitors on blood pressure in depression treatment: outcomes from placebo and serotonin and noradrenaline reuptake inhibitor controlled trials. Neuropsychiatr Dis Treat 2017; 13: 2781-96.

44 Mendelson N, Gontmacher B, Vodonos A, Novack V, Abu-AjAj M, Wolak A, et al. Benzodiazepine consumption is associated with lower blood pressure in ambulatory blood pressure monitoring (ABPM): retrospective analysis of 4938 ABPMs. Am J Hypertens 2018; 31: 431-7.

45 Latvala A, Kuja-Halkola R, Rück C, D'Onofrio BM, Jernberg T, Almqvist C, et al. Association of resting heart rate and blood pressure in late adolescence with subsequent mental disorders: a longitudinal population study of more than 1 million men in Sweden. JAMA Psychiatry 2016; 73: 1268-75.

46 Thayer JF, Friedman BH, Borkovec TD. Autonomic characteristics of generalized anxiety disorder and worry. Biol Psychiatry 1996; 39: 255-66.

47 Gonçalves R, Rodrigues H, Novaes F, Arbol J, Volchan E, Coutinho ESF, et al. Listening to the heart: a meta-analysis of cognitive behavior therapy impact on the heart rate of patients with anxiety disorders. J Affect Disord 2015; 172: 231-40.

48 Gariepy G, Nitka D, Schmitz N. The association between obesity and anxiety disorders in the population: a systematic review and meta-analysis. Int J Obes 2010; 34: 407-19.

49 Barry D, Pietrzak RH, Petry NM. Gender differences in associations between body mass index and DSM-IV mood and anxiety disorders: results from the National Epidemiologic Survey on Alcohol and Related Conditions. Ann Epidemiol 2008; 18: 458-66.

50 Gibson-Smith D, Bot M, Brouwer IA, Visser M, Penninx BW. Diet quality in persons with and without depressive and anxiety disorders. J Psychiatr Res 2018; 106: 1-7.

51 McCaffery JM, Niaura R, Todaro JF, Swan GE, Carmelli D. Depressive symptoms and metabolic risk in adult male twins enrolled in the National Heart, Lung, and Blood Institute twin study. Psychosom Med 2003; 65: 490-7. 
52 Skilton MR, Moulin P, Terra J-L, Bonnet F. Associations between anxiety, depression, and the metabolic syndrome. Biol Psychiatry 2007; 62: 1251-7.

53 Herva A, Räsänen P, Miettunen J, Timonen M, Läksy K, Veijola J, et al. Cooccurrence of metabolic syndrome with depression and anxiety in young adults: the Northern Finland 1966 Birth Cohort Study. Psychosom Med 2006 68: 213-6.

54 van Milligen BA, Lamers F, Guus T, Smit JH, Penninx BW. Objective physical functioning in patients with depressive and/or anxiety disorders. J Affect Disord 2011; 131: 193-9.

55 Williams LJ, Pasco JA, Jacka FN, Hodge JM, Kotowicz MA, Berk M. Quantitative Heel Ultrasound (QUS) measures of bone quality in association with mood and anxiety disorders. J Affect Disord 2013; 146: 395-400.

56 Seldenrijk A, van Hout HP, van Marwijk HW, de Groot E, Gort J, Rustemeijer C, et al. Depression, anxiety, and arterial stiffness. Biol Psychiatry 2011; 69: 795803.

57 Yeragani VK, Kumar R, Bar KJ, Chokka P, Tancer M. Exaggerated differences in pulse wave velocity between left and right sides among patients with anxiety disorders and cardiovascular disease. Psychosom Med 2007; 69 717-22.

58 Bonnet F, Irving K, Terra J-L, Nony P, Berthezène F, Moulin P. Anxiety and depression are associated with unhealthy lifestyle in patients at risk of cardiovascular disease. Atherosclerosis 2005; 178: 339-44.

59 Vogelzangs N, Beekman A, De Jonge P, Penninx B. Anxiety disorders and inflammation in a large adult cohort. Transl Psychiatry 2013; 3: e249-e.
60 Michopoulos V, Powers A, Gillespie CF, Ressler KJ, Jovanovic T. Inflammation in fear-and anxiety-based disorders: PTSD, GAD, and beyond. Neuropsychopharmacology 2017; 42: 254-70

61 Black C, Bot M, Scheffer P, Penninx B. Oxidative stress in major depressive and anxiety disorders, and the association with antidepressant use; results from a large adult cohort. Psychol Med 2017; 47: 936-48.

62 Beaudreau SA, O'Hara R. Late-life anxiety and cognitive impairment: a review. Am J Geriatr Psychiatry 2008; 16: 790-803.

63 Davis K, Hotopf M. Mental health phenotyping in UK Biobank. Prog Neurol Psychiatry 2019; 23: 4-7.

64 Glanville KP, Coleman JR, Howard DM, Pain O, Hanscombe KB, Jermy B, et al. Multiple measures of depression to enhance validity of Major Depressive Disorder in the UK Biobank. BJPsych Open 2021; 7: e44.

65 Bandelow B, Michaelis S. Epidemiology of anxiety disorders in the 21st century. Dialogues Clin Neurosci 2015; 17: 327-35.

66 Fry A, Littlejohns TJ, Sudlow C, Doherty N, Adamska L, Sprosen T, et al. Comparison of sociodemographic and health-related characteristics of UK Biobank participants with those of the general population. Am J Epidemiol 2017; 186: 1026-34.

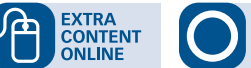
OPEN

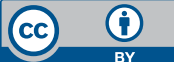

\section{psychiatry in television}

\section{Social isolation and alcohol use: lessons from The Queen's Gambit}

\section{Faraan Cheema (iD) and Paul Wilkinson}

The Queen's Gambit is a 2020 Netflix miniseries that follows Beth Harmon, a fictional orphan chess prodigy battling to become the world's best player while simultaneously struggling with alcohol and drug dependency. Although some have argued that the depiction of Beth's recovery from addiction is unrealistic, these criticisms fail to acknowledge the detailed presentation of the factors that lead to Beth's substance use and subsequent sobriety.

Throughout the series, Beth's substance use has consistent triggers: shame, anxiety and isolation. These three factors combine to create a perfect storm following Beth's second defeat to World Champion Vasily Borgov. Regarding shame, Beth's defeat damages her self-image, which depends heavily on being the best. The damage to her self-confidence also results in feelings of anxiety due to her upcoming rematch against Borgov. Her subsequent alcohol use can be seen as an avoidant coping mechanism to deal with these feelings of shame and anxiety. Beth demonstrates features of vulnerable narcissism, and shame can indeed mediate the effects of vulnerable narcissism on addiction. These feelings cause Beth to shun the chess players who previously supported her, leading to social isolation. These problems spiral, leading to Beth's most serious period of dependence yet.

Beth's recovery stems from several events that resolve these three factors. Seeing the accomplishments of Jolene and Annette, characters who lack Beth's natural talent but still have worked hard to meet their goals, leads Beth to realise that her alcohol use is wasting her gift. Subsequently Beth sees that Mr Shaibel (the janitor who first taught Beth to play chess) has a wall of newspaper cuttings dedicated to her entire chess career, and she breaks down in tears. The pride shown by Mr Shaibel affects Beth in two ways: it adds to the feeling that her alcohol use is wasting her talents, but is also crucial for the restoration of her positive self-image. This allows her to overcome her feelings of shame and anxiety and once again be driven by a desire to win. The crux of Beth's recovery is support from Jolene - throughout the series Beth consistently manages to maintain long periods of sobriety as long as she is not alone. While Beth's alcohol cravings continue under the stress of the final, the support from Beth's fellow chess players is shown to be crucial for her maintained sobriety and ultimate victory.

In summary, Beth's recovery is not unrealistic, but rather is consistent with the resolution of many of the factors that led to Beth's substance use. A fairer judgement would be that Beth's addiction is less representative of those with more severe problems, and a merit of this presentation of substance use is that it may be relatable to more viewers. Watching The Queen's Gambit can give viewers an opportunity to reflect on how isolation may affect their own substance use, and reinforces the key protective role of social support.

(c) The Author(s), 2022. Published by Cambridge University Press on behalf of the Royal College of Psychiatrists 